\title{
Repli sur soi et ouverture vers l'autre dans l'Afrique du Sud contemporaine
}

Représentation(s) de la mobilité et insertion des migrants sénégalais et maliens à Johannesburg

Withdrawing into oneself and opening up to others in contemporary South

Africa. Representation(s) of mobility and integration of Senegalese and Malian

migrants in Johannesburg

Christine Ludl

\section{OpenEdition}

1 Journals

Édition électronique

URL : http://journals.openedition.org/transcontinentales/790

DOI : 10.4000/transcontinentales.790

ISBN : 978-2-7351-1557-0

ISSN : $1775-397 X$

Éditeur

Editions de la maison des sciences de l'homme

Édition imprimée

Date de publication : 31 décembre 2010

ISSN : 1950-1684

\section{Référence électronique}

Christine Ludl, «Repli sur soi et ouverture vers l'autre dans I'Afrique du Sud contemporaine »

Transcontinentales [En ligne], 8/9 | 2010, document 5, mis en ligne le 31 décembre 2010, consulté le 08 septembre 2020. URL : http://journals.openedition.org/transcontinentales/790 ; DOI : https://doi.org/ 10.4000/transcontinentales.790

Ce document a été généré automatiquement le 8 septembre 2020

Tous droits réservés 


\section{Repli sur soi et ouverture vers l'autre dans l'Afrique du Sud contemporaine}

Représentation(s) de la mobilité et insertion des migrants sénégalais et maliens à Johannesburg ${ }^{1}$

Withdrawing into oneself and opening up to others in contemporary South Africa. Representation(s) of mobility and integration of Senegalese and Malian migrants in Johannesburg

Christine Ludl

\section{Migrations africaines et transformations urbaines à Johannesburg}

1 Après la fin de l'apartheid, les premières élections démocratiques en 1994, l'ouverture des frontières et l'abolition de la législation restrictive qui avait perfectionné la ségrégation (spatiale) des différents groupes, l'Afrique du Sud est devenue une destination majeure pour des migrants internes ou venant du reste du continent ${ }^{2}$. Ces migrants transitent ou s'installent le plus souvent dans les quartiers centraux des grandes villes - notamment à Johannesburg -, qui étaient exclusivement réservés à la population «blanche » sous l'apartheid et sont aujourd'hui habités par une population d'origine très diverse (Morris 1994, 1999). Ces quartiers déshérités sont souvent marqués par l'insécurité, mais aussi par la reconstruction urbaine et les débuts d'un processus de gentrification ${ }^{3}$. Les migrants qui y habitent et $y$ travaillent disent souvent faire l'objet d'attaques, de vols et de discriminations à caractère raciste de la part des résidents sud-africains «noirs", de la police et d'autres groupes de migrants. Si ces violences sont bien documentées depuis les années 1980 (Bekker et al. 2008; Wa KabweSegatti 2008: 106), elles ont pris une ampleur et une médiatisation inédites avec les émeutes de mai 2008 qui ont visé majoritairement des étrangers ${ }^{4}$. 
2 Les études qui visent à expliquer ces événements relèvent d'un caractère « hétéroclite " (Wa Kabwe-Segatti 2008: 100). Elles ont souvent avancé des raisons économiques globales et locales, notamment un taux de chômage croissant ${ }^{5}$ et des frustrations devant le manque ou la lenteur d'accès aux services de base, un contrôle de frontières inefficace, une "culture de la violence» ou un accroissement des sentiments « xénophobes » (Sharp 2008a, 2008b; SAIRR 2008; HSRC 2008).

3 Cependant, ces explications laissent un certain nombre de questions ouvertes. Ainsi, l'Afrique du Sud connaît des inégalités économiques et sociales importantes. On peut faire l'hypothèse de la continuité d'un projet de prospérité matérielle de société allant de l'apartheid à la période postapartheid (Neocosmos 2006). Néanmoins, les lieux des violences ne correspondent pas aux zones où l'on trouve les populations les plus démunies ou le taux le plus élevé de migrants (Wa Kabwe-Segatti 2008 : 106 ; Wa KabweSegatti et Fauvelle-Aymar 2009). En outre, historiquement, le recours à la violence dans la gestion des conflits sociaux et politiques a joué un rôle important en Afrique du Sud (Beinart 1992; voir Wa Kabwe-Segatti 2008: 99), mais l'argument culturaliste d'une " culture de la violence » est loin de saisir la complexité du phénomène.

4 En ce qui concerne l'argument d'un accroissement des sentiments "xénophobes ", de nombreuses études, menées avant et après les événements de mai 2008, ont démontré l'augmentation du ressentiment et des violences envers les étrangers (Palmery 2002 ; Idasa 2008 ; Crush et al. 2008 ; Mattes et al. 1999). Cependant, ce constat ne suffit pas à expliquer les violences. Des recherches récentes ont révélé le rôle crucial des pouvoirs locaux dans la mobilisation - et parfois dans le contrôle - de la violence (Misago et al. 2009). En outre, si des personnes déplacées en mai 2008 ont souvent identifié leurs voisins parmi les agresseurs, elles disent avoir entretenu de bonnes relations avec eux jusque-là ${ }^{6}$. Dans une perspective similaire, on peut identifier un " compromis fragile " entre commerçants étrangers et sud-africains au marché de Yeoville, un quartier du centre-est de Johannesburg, caractérisé par un haut pourcentage de migrants africains, notamment congolais ${ }^{7}$. Ainsi, ces recherches pointent vers une question importante qui n'a pas été explorée jusqu'ici : celle des interactions quotidiennes entre migrants et Sud-Africains, et des représentation(s) ${ }^{8}$ mutuelles des deux groupes. À cela s'ajoute que la plupart des études se sont concentrées sur les townships et les zones urbaines informelles où les émeutes ont eu lieu et ont souvent mis l'accent sur l'explication des facteurs qui ont déclenché les événements. En conséquence, on ne sait que très peu de choses sur les relations et les perceptions mutuelles entre migrants et citoyens sudafricains dans les centres-villes.

5 Je propose ici de contribuer à éclaircir cette question à partir des résultats d'une enquête sur les imbrications entre les représentation(s) de la mobilité et de la réussite et les modes d'insertion dans la ville des migrants originaires de la vallée du fleuve Sénégal (Mali et Sénégal) ${ }^{9}$ et d'autres régions du Sénégal dans le centre-ville de Johannesburg ${ }^{10}$. Alors que les derniers sont souvent organisés dans des confréries musulmanes, notamment dans les deux principales confréries du Sénégal, la Muridiyya et la Tijaniyya, les premiers font moins souvent partie d'organisations religieuses. Leurs projets migratoires suivent surtout des logiques familiales (Timera 1996; Fall $2003: 17$; Tall $2002: 549$ ) alors que ceux des mourides se caractérisent par une relation étroite entre voyage, religion et commerce (Diouf 2000).

6 Plus précisément, dans mon enquête, on distingue trois groupes de migrants: premièrement, deux familles (au sens large) de Maliens originaires de la région de 
Kayes, qui tiennent une boutique et plusieurs cybercafés à Yeoville. Ils résident soit dans le quartier, soit dans les environs, notamment à Ellis Park. Si certains de ces migrants se sont installés en Afrique du Sud il y a cinq ou six ans, la majorité d'entre eux sont des jeunes qui viennent d'arriver. La plupart d'entre eux travaillent dans le commerce et enchaînent des emplois temporaires. Le deuxième cas est fourni par un groupe de migrants sénégalais qui font partie d'une branche de la Tijaniyya, la deuxième confrérie du Sénégal. Certains de ces migrants sont arrivés à Johannesburg dans la deuxième moitié des années 1990, d'autres sont là depuis quelques années. Plusieurs d'entre eux habitent à Troyeville, à Bezuidenhout Valley ou dans les quartiers environnants. Ils y tiennent des boutiques de bijoux, de vêtements, de matériel informatique ou des cybercafés; d'autres sont informaticiens, mécaniciens, couturiers ou grossistes. Certains sont mariés à des femmes sud-africaines ou ont fait venir leur famille en Afrique du Sud, mais la plupart d'entre eux sont mariés au Sénégal. Le troisième exemple est constitué par un groupe de migrants sénégalais faisant partie de la confrérie mouride. Ils étaient parmi les premiers migrants sénégalais arrivés en Afrique du Sud après la fin de l'apartheid. Ils sont souvent des grossistes qui voyagent régulièrement en Asie pour acheter du stock ou travaillent dans le commerce d'objets d'art sur les marchés touristiques des quartiers aisés du nord de Johannesburg, comme Rosebank ou Sandton, au Chameleon Village Market, situé environ à $100 \mathrm{~km}$ de Johannesburg sur la route touristique menant aux Magaliesberge, ou au Bruma Lake Flea Market, à l'est de Johannesburg ${ }^{11}$. Ceux qui ont pu établir leur propre commerce ont quitté les quartiers du centre-ville comme Yeoville ou Hillbrow pour les quartiers aisés du nord. Dans la plupart des cas, leur situation économique est relativement confortable et ils considèrent qu'ils ont vécu la success story des Sénégalais en Afrique du Sud en passant du statut de petits vendeurs de rue à celui de grossistes ou de propriétaires d'un commerce sur les marchés touristiques (Moustapha, entretien, 12 novembre 2008, Rosebank).

7 Je monterai par la suite comment, pour chacun de ces trois groupes, les représentation(s) de la migration et de la réussite et les modes d'insertion dans la ville interagissent et influent sur les stratégies migratoires et les projets d'avenir des migrants. Je montrerai qu'il n'existe pas de réponse univoque à cette question et qu'elle dépend largement du type de réseaux sociaux dans lesquels les migrants sont insérés. Mais avant, j'expliquerai brièvement le concept théorique de représentation(s) sur lequel je m'appuie ainsi que les principales caractéristiques des représentation(s) de la mobilité et du succès et des modes d'insertion dans la ville des migrants.

\section{Les représentation(s) de la migration et de la réussite}

8 Les représentation(s) de la migration et du voyage se construisent dans un système complexe d'aspirations, de possibilités et de contraintes, dans un processus dynamique auquel participent tant les représentation(s) de la réussite au sein de la société d'origine que les expériences des migrants dans les pays de destination ou de transit, et les représentation(s) qu'ils s'en font. À cela s'ajoute leur flexibilité. Cassirer, philosophe de la culture, conçoit une "élasticité» (1999a: 106) et une "direction» (Richtungscharakter) des représentation(s) qui «renvoie au-delà de l'Ici et du Maintenant " (Cassirer 2002: 232, ma traduction; Sandkühler 1999: 1385). Cette élasticité leur confère la capacité de s'étendre dans des contextes et des 
environnements différents ; ce qui change, c'est le regard que l'on porte dessus ; on les voit dans une autre perspective, à partir d'un autre " point de vue " (Blickpunkt) ce qui leur donne une autre signification. Dans une perspective similaire, Octave Mannoni, à partir de l'expérience d'une contradiction entre réalité et imaginaire, démontre comment plusieurs possibilités peuvent coexister sans entrer en conflit. Sa fameuse phrase «je sais bien, mais quand même» (Mannoni 1969: 11) condense ces versions d'une coexistence paisible entre imaginaire et réalité. Comme chez Cassirer, ce qui rend cette coexistence possible, c'est une transformation de l'imaginaire initial qui lui confère une signification différente ${ }^{12}$.

9 Dans le cas des projets migratoires, ces représentation(s) renvoient à celles de la réussite et du prestige social et aux voies légitimes pour y accéder. Elles s'établissent en fonction de ce que les migrants pensent que les sociétés d'origine - et d'autres - leur offrent pour réaliser leurs aspirations. Elles constituent des imaginaires dans le sens d'une possibilité et d'un idéal ${ }^{13}$ que les migrants cherchent à atteindre et d'un ensemble amovible que les migrants reconsidèrent, réévaluent et adaptent à chaque étape de leur voyage. Alors que la majorité des migrants affirment partir pour gagner de l'argent, pour soutenir la famille au pays et pour pouvoir y construire leur propre existence, les représentation(s) et les imaginaires de la réussite s'avèrent plus complexes. Comme le résume Zékéria Ould Ahmed Salem, ils concernent "l'univers des itinéraires d'individus ou de groupes en quête de survie ou d'une réussite sociale prestigieuse [...] d'acteurs préoccupés d'arracher une place jugée digne dans une société et un État en proie à des transformations parfois vertigineuses [...] où l'obsession de la survie est tout à la fois matérielle et sociale, politique, symbolique et même psychologique " (2001: 78).

Plus concrètement, les représentation(s) de la réussite et de la migration renvoient à deux tensions : d'une part, celle entre les objectifs matériels et ceux de la découverte et du savoir acquis par le voyage et, d'autre part, à une tension entre les logiques collectives et les objectifs individuels du projet migratoire. Ces deux tensions forment un équilibre fragile où le poids des différents éléments est constamment ajusté. Ainsi, des ressources monétaires pour soutenir la famille, mais aussi des biens de consommation de marque en tant que signes extérieurs de la réussite confèrent $\mathrm{du}$ prestige social. En même temps, confronter et vaincre les difficultés de la migration et acquérir des expériences et du savoir contribuent à ce prestige et, au retour, ouvrent aux migrants l'accès à une position sociale plus élevée ${ }^{14}$. En même temps, les migrants cherchent parfois aussi à s'éloigner de la famille pour atténuer les contraintes sociales et réaliser des projets individuels. Comme le soulignent Fariba Adelkhah et JeanFrançois Bayart, «le voyage est souvent vécu comme un style de vie picaresque ou épique, même lorsqu'il répond à des contraintes structurelles ou conjoncturelles, d'ordre économique ou politique, et il s'apparente à une expérience morale de la subjectivation qui en est l'une des motivations principales » $(2007: 9)^{15}$.

11 D'un côté, je prends ces représentation(s) de la réussite comme point de départ. D'autre part, ces mêmes représentation(s) se transforment en fonction des spécificités du contexte social, politique et économique des pays «d'accueil » et des expériences que les migrants y font. C'est dans cette perspective que l'insertion des migrants dans la ville entre en jeu. 


\section{L'insertion dans la ville}

12 Je comprends ici, dans un premier temps, la notion de l'insertion dans la ville dans le sens large de relations à des institutions, à des personnes et à l'espace, pour montrer, dans un deuxième temps, comment les imbrications entre relations sociales et relation à l'espace renouent avec les notions de placemaking (Massey 1994, 1995) et de people as infrastructure (Simone 2008) et comment celles-ci peuvent être complétées par la notion de représentation(s). Tout d'abord, les relations des migrants aux institutions concernent surtout l'administration sud-africaine, notamment le Department of Home Affairs, chargé de la distribution des titres de séjour et, dans la vie quotidienne, la police ; les interactions avec cette dernière se caractérisent souvent par la corruption, des harcèlements et la violence (Vigneswaran et Duponchel 2009). À cet égard, les migrants sénégalais et maliens semblent s'en sortir plutôt bien, ce qu'ils attribuent à l'image dont ils jouissent dans l'opinion publique, image plus positive que celle d'autres groupes de migrants. Pour la même raison, ceux qui sont obligés de renouveler régulièrement leur permis de séjour ne rencontrent, dans la plupart des cas, pas de difficultés majeures (entretiens du 2 avril 2009, Yeoville, et du 23 mai 2010, marché touristique près de Johannesburg). Cependant, il s'agit ici d'une minorité. La majorité des migrants sénégalais et maliens déposent à leur arrivée une demande d'asile politique pour obtenir une carte de demandeur d'asile de trois mois renouvelables qui leur permet de circuler librement dans le pays et de disposer de papiers en règle lors des contrôles de police. Cependant, ils n'ont pas droit au statut de réfugié. Une fois leur demande rejetée, pour éviter de tomber en situation illégale, de nombreux migrants sénégalais, en particulier au sein de la confrérie mouride, s'engagent dans des mariages ou des concubinages (life partnership) avec des résidantes sud-africaines pour accéder à un titre de séjour stable ou à la nationalité sud-africaine. $75 \%$ des Mourides seraient mariés à des femmes sud-africaines et environ $30 \%$ possèderait la nationalité sudafricaine (Moustapha, entretien, 12 novembre 2008, Rosebank) ${ }^{16}$. Ces mariages renvoient au deuxième mode d'insertion dans la ville, les relations à des personnes. En dehors des mariages, le travail est un lieu important de ces relations, notamment quand les migrants reçoivent des clients dans leurs magasins ou quand ils y emploient des Sud-Africains. La plupart des migrants choisissent de créer leur propre business, car il leur est souvent difficile de trouver un emploi et d'être bien payés. Comme le confirme un migrant, "il faut créer son propre business parce que les Sud-Africains n'embauchent pas les étrangers» (Mamadou, entretien, 7 mai 2010, СвD). Un autre souligne que « tu peux avoir l'argent, mais il faut avoir aussi des papiers. Il faut avoir un bon papier d'ici pour qu'ils vont te payer bien quoi [...] C'est le problème qui est là » (Malik, entretien, 12 août 2009).

13 À cela s'ajoute l'insertion dans des réseaux de ressortissants sénégalais et maliens, notamment des réseaux familiaux et les confréries musulmanes. Celle-ci s'avère cruciale dans un contexte où la taille du groupe - par rapport à d'autres groupes de migrants dans le même pays et par rapport aux autres destinations des Sénégalais et des Maliens en Afrique, en Europe ou aux États-Unis - est relativement petite; on estime entre 4000 et 5000 , le nombre de Sénégalais en Afrique du Sud ${ }^{17}$. En ce qui concerne les Maliens, il n'existe pas de données fiables sur leur nombre en Afrique du Sud, mais celui-ci est certainement bien inférieur à celui des Sénégalais. Selon un document de l'Organisation internationale des migrations (ОIM), en 2007, 6,1\% de tous 
les Maliens à l'étranger qui ont déposé une demande d'asile au cours de l'année l'ont fait en Afrique du Sud ce qui revient à 87 personnes (Ballo $2009: 118)^{18}$. Les Maliens que j'ai rencontrés affirment que "tous les Maliens à Johannesburg se connaissent" (Dramé, entretien, 26 mars 2009, Yeoville). De tels propos sont difficiles à vérifier ; j'ai cependant constaté que les mêmes éléments de discours, comme celui sur la colonisation (voir plus bas), revenaient systématiquement dans les entretiens avec les membres des deux groupes ce qui renvoie, en effet, à un groupe relativement restreint au sein duquel certains thèmes font l'objet de discussions et d'interprétations spécifiques.

On verra que les modes d'insertion dans la ville des migrants dépendent dans une large mesure de la diversité sociale de ces réseaux. Ainsi, les confréries regroupent des migrants originaires de différentes régions du Sénégal ou bien elles se sont ouvertes à des migrants d'autres pays africains ou à des Sud-Africains. Contrairement à cela, les réseaux familiaux s'avèrent culturellement et socialement plus homogènes. On note l'absence d'autres associations de migrants telles que les associations villageoises des migrants originaires de la vallée du fleuve Sénégal. Comme le confirme un migrant malien, «en France, les migrants se regroupent beaucoup plus. Ici c'est plus individuel » (conversation, 6 avril 2009, Yeoville). Un membre de la Tijaniyya rapporte qu'au sein de la dahira (associations de disciples), on réfléchissait à fonder une association, mais que finalement on ne voyait pas l'intérêt de créer une double structure (entretien, 7 mai 2010, CBD).

Troisièmement, les relations interpersonnelles influent directement sur la relation à l'espace. Celle-ci prend une importance particulière en Afrique du Sud où la mobilité de personnes a été fortement stigmatisée pendant l'apartheid et fait l'objet de contrôles arbitraires et extralégaux (Landau 2010: 218) ${ }^{19}$. En conséquence, comme Achille Mbembe l'a fait remarquer, "most studies of Johannesburg have read the city as nothing but the spatial embodiment of unequal economic relations and coercive and segregationist policies» (Mbembe et Nuttall 2004 : 353). Ceci vaut tant pour la « [l]ong tradition on urban inquiry that focuses on the spatial dislocation, the class differentiation, and the racial polarization imprinted on the urban landscape by apartheid state-sanctioned segregation and planning " (Mbembe et Nuttall 2004: 356) que pour la période postapartheid. Ici, prévaut le paradigme du « développement urbain » qui traite de la restructuration spatiale de la ville per se et qui interroge avant tout la question du changement de la ville par rapport à la gouvernance institutionnelle, la déracialisation de l'accès aux services et la politique locale (Mbembe et Nuttall $2004: 358$; Bremner et Subirós 2007 ; Murray 2008).

\section{Transformation de représentation(s) et stratégies migratoires}

16 Pour chacun des trois groupes concernés ici, comment s'articulent, d'une part, la question des représentation(s) de la migration et de la réussite - concrètement, la tension entre les objectifs monétaires et éducatifs de la migration - et, d'autre part, la question de l'insertion dans la ville, c'est-à-dire, les représentation(s) et interactions des migrants avec leur environnement social et spatial? Quels sont les différents projets d'avenir et stratégies migratoires qui en découlent? On verra que ces articulations et transformations dépendent des modes d'insertion des migrants dans des réseaux sociaux, mais également de la situation des migrants dans le parcours 
migratoire (âge, durée du séjour, situation socioéconomique). Plus important encore, c'est la notion de la diversité qui se trouve au centre de ces transformations ${ }^{20}$.

\section{«C'est un pays qui est beaucoup raciste. C'est un pays de métis quoi !»}

17 Tout d'abord, en ce qui concerne les migrants maliens et leurs objectifs migratoires, les plus âgés et les plus établis mentionnent parfois les objectifs de la découverte et du savoir par le voyage. Comme le constate un migrant, «you are learning a lot of things and you see something [...] You can know something you didn't even know before» (Dramé, entretien, 26 mars 2009, Yeoville). Mais la majorité valorise les bénéfices monétaires. Ainsi, après avoir raconté qu'il a arrêté ses études, un jeune Malien affirme «qu'on a trop l'argent dans la tête " et un autre constate que "nous, les Maliens, on veut beaucoup d'argent, très vite » (conversations le 25 mars et le 6 avril 2009, Yeoville). Ceci confirme mes recherches antérieures sur les représentation(s) de la réussite et de la migration de jeunes Sénégalais qui veulent réaliser des objectifs de haut de gamme dans des délais très courts en contournant toute phase d'apprentissage et d'efforts. La nouveauté de ces représentation(s) réside dans leur contraste avec les représentation(s) de la réussite antérieures qui valorisaient l'éducation et le savoir ou, plus récemment au sein du mouvement buul faale, le travail et les efforts individuels (Ludl 2008b; sur le mouvement buul faale voir Havard 2001). À Johannesburg, ils se plaignent que «ça va un peu, un peu seulement », que « le travail ici ne rapporte pas assez », « seulement 200 \$» et que le «business ici est trop petit» (conversation le 26 mars 2009, Yeoville). Cependant, ceux qui sont arrivés il y a quelques années et qui ont pu trouver un travail ou établir un commerce considèrent que l'Afrique du Sud offre de bonnes conditions pour réaliser des bénéfices économiques (par exemple, entretiens le 26 mars 2009 et le 12 août 2009).

18 Quant à la question de savoir comment les Maliens perçoivent et se lient à leur environnement social et spatial, leurs discours reflètent souvent l'hostilité des SudAfricains à leur égard. D'une manière générale, ils considèrent qu' « ils n'aiment pas les étrangers ici » (Alioune, conversation, 25 mars 2009, Yeoville) et deux jeunes Maliennes appartenant à la même famille, l'une arrivée il y a cinq mois, l'autre huit ans auparavant, trouvent l'Afrique du Sud « compliquée [...] On dirait que nous ne sommes pas des Africains» (Mamo et Kandiétou, conversation, 25 mars 2009, Yeoville). Plus précisément, la criminalité est omniprésente dans leurs récits - et leurs soucis sont réels : «Bon, en fait, il y a trop de bandits quoi. Il y a trop de bandits [...] Toujours on a [des] problèmes avec eux, ici [...] Oui. C'est le problème qui est là. Le problème c'est le crime [sic !] [...] [Il élève la voix] C'est que je t'ai dit! Même pas trois semaines, ils ont tiré sur un autre papa ici. Il est décédé. Donc, vraiment, je suis tellement découragé d'ici quoi. Je veux pas rester ici. Je vais voir en Europe » (Malik, entretien, 12 août 2009).

Selon les Maliens, à part des références à la religion et au contrôle social au sein du groupe, deux raisons expliquent ce taux élevé de criminalité en Afrique du Sud. D'une part, ils font une distinction entre les pays qui ont été colonisés par la France et ceux qui l'ont été par l'Angleterre. Alors que les premiers respectaient les droits de l'homme, les seconds n'avaient aucune idée de cette notion - d'où le niveau élevé de criminalité et d'atteintes à la vie des personnes. «Vraiment, nous, je peux dire que c'est tous les pays que l'Angleterre a colonisés quoi, ils ont des problèmes, ils n'ont pas l'intelligence. 
Tu vois ?! Nous, au moins, au fait, la France nous a colonisés, la France est tellement intelligente, donc nous aussi, on est intelligent. Chez nous, en Afrique de l'Ouest, tu peux agresser quelqu'un, tu vas prendre tout son truc, mais tu vas jamais le tuer quoi. C'est le problème qui est là quoi [...] Ils sont bêtes, ils n'ont pas l'intelligence, ils connaissent pas les Droits de l'Homme, tu vois, ils prennent l'homme comme des poulets, des chiens quoi, comme on tue des chiens quoi, du poulet, alors que c'est pas bon. Un homme là, vraiment, il faut [le] respecter » (Malik, entretien, 12 août 2009).

D'autre part, ils attribuent la criminalité, qu'ils associent étroitement au racisme, à la diversité sociale de la société sud-africaine. "Oui, donc c'est pourquoi il y a beaucoup de crime quoi. Parce qu'ici c'est un pays vraiment, c'est un pays qui est beaucoup raciste. C'est un pays de métis quoi ! De blancs et de noirs, et de métis, et des Indiens. Tout le monde est mélangé ici, chacun dans son chacun. Les noirs sont à part, les blancs sont à part, les Indiens sont à part. C'est pourquoi il y a trop de crimes quoi. Vraiment. Les noirs n'aiment pas les Indiens, ils n'aiment pas aussi les blancs, les blancs aussi n'aiment pas les noirs, les Indiens aussi n'aiment pas les blancs. Vraiment c'est pourquoi c'est le grand problème d'ici quoi » (Malik, entretien, 12 août 2009).

Le sentiment d'insécurité - et l'expérience concrète de celle-ci - détermine largement la vie quotidienne et les stratégies sociales et spatiales des migrants maliens. Ils disent « socialiser avec les autres francophones dans le quartier », mais restent la plupart du temps entre eux, c'est-à-dire, en famille. S'ils ne travaillent pas (ailleurs), ils passent la journée à la boutique ou au cybercafé où ils prennent les repas qu'une femme de la même famille prépare et, aux heures de prière, ils partent ensemble à la mosquée située à quelques centaines de mètres dans la même rue. Ainsi, ils limitent leurs relations sociales au minimum et à des personnes de confiance. Ceci vaut également pour leurs relations à l'espace de la ville. Les principaux trajets des migrants sont entre leurs maisons et leurs lieux de travail, situés souvent dans le même quartier ou dans un quartier environnant. L'un deux constate qu' «avec les bandits, tu peux pas sortir. Tu quittes au travail seulement, tu es à la maison » (Malik, entretien, 12 août 2009, Ellis Park). Un autre migrant qui n'habite qu'à un kilomètre de son lieu de travail, raconte « when I leave at home at 9 o'clock, then I come here; so when I just enter here, I don't go anywhere till we are closing. So, I go home directly, I go to watch TV [...] I don't go anywhere » (Dramé, entretien, 26 mars 2009).

Comme ces propos l'indiquent, les migrants se déplacent parfois dans des espaces extrêmement limités. C'est par exemple le cas d'un migrant sénégalais qui passe l'essentiel de ses journées dans un périmètre de quelques centaines de mètres : il loue une chambre juste à côté de son lieu de travail à Yeoville, il appelle sa famille au Sénégal d'un "télécentre " dans une rue latérale de la rue principale du quartier et achète à manger et tout dont il a besoin au marché situé juste à côté. Les seuls déplacements qui interrompent cette routine quotidienne sont ses visites au CBD pour se procurer du stock et au Department of Home Affairs pour renouveler son permis de séjour. Il justifie la double stratégie sociale et spatiale qu'utilisent de nombreux migrants pour contrôler le risque d'attaques et de vols ainsi : "To visit someone you don't know is very dangerous, because you don't know the people [...] Somewhere you don't know, is dangerous. Ne? Somewhere you don't know is very dangerous. If it was West Africa, you know, out there, is not so dangerous. [There are] no criminals throughout West Africa. Ya, so, but here, [it's] not the same» (Seydou, entretien du 3 avril 2009, Yeoville). Ces propos confirment le besoin de maintenir constamment un état de hyperawareness et de preparedness, ainsi 
que "la texture d'un espace social extrêmement fragmenté » du centre-ville de Johannesburg dont parle Abdoumaliq Simone. "Thus, the inner city has a complex geography that residents must navigate according to a finely tuned series of movements and assumptions. There are places where they know they must no go or be seen but this knowledge often depends on highly variable notions about which places are safe and which are not" (Simone $2008: 76,82$ et 85).

Comme on l'a vu dans un des extraits d'entretien, la plupart des Maliens préfèrent aller ailleurs, surtout en Europe. Ils mobilisent des connaissances au Mali, en France ou dans d'autres pays européens pour qu'elles les soutiennent dans leurs démarches ou, comme Malik, essayent de regagner la France en traversant la Méditerranée. Malgré une tentative échouée, il est déterminé à tenter encore une fois sa chance. Il confirme qu'il "rêve beaucoup [de] là-bas! Je rêve vraiment, il faut que je voie là-bas quoi" (entretien, 12 août 2009, Ellis Park). «Parce que l'Afrique c'est la merde! Il faut que je parte!» (conversation téléphonique, 5 août 2009). Malgré «la crise qui frappe maintenant l'Europe ", Malik pense que c'est "la vie facile ", « une vie moins chère " qui, en raison de la valeur de la monnaie, lui permettrait de construire facilement un commerce et une maison pour sa famille en Afrique et de fonder sa propre famille (Malik, entretien, 12 août 2009, Ellis Park). Sous la pression sociale apparaît l'envie de partir en Europe qui reste la destination la plus prestigieuse. Cette question du prestige des destinations se confirme, car l'Afrique du Sud est le pays le plus valorisé en Afrique. "Aujourd'hui nous sommes en Afrique du Sud, c'est un bon pays, c'est une bonne capitale, surtout Johannesburg, c'est une bonne capitale, ici en Afrique. Oui. C'est la plus grande capitale ici en Afrique, hein ? [Rires] Ah oui. C'est ça [...] Pour moi. Pour moi en tout cas. Pour moi, c'est la plus grande capitale qu'on peut comparer comme en Europe, comme en Amérique. C'est le seul pays qui est en Afrique où on peut présenter bien partout. Oui. [Rires] Oui, c'est ça " (Malik, entretien, 12 août 2009, Ellis Park, je souligne). Néanmoins, l'Afrique du Sud a un prestige inférieur à celui des pays européens. Ils considèrent «qu'il faut quitter l'Afrique » (Moussa, conversation, 6 avril 2009, Yeoville) et les propos de Malik révèlent encore une fois le stigma social d'être en Afrique. "Mon frère, lui il est à Paris, il est parti, en ce moment j'avais l'âge de 14 ans. Et tous mes grands frères sont là-bas et je suis le seul en Afrique ici. Et comment je vais faire, je n'ai pas eu la chance de partir » (entretien, 12 août 2009, Ellis Park). Ainsi, toutes leurs aspirations non réalisées se condensent alors dans la formule « on est venu, mais on n'a rien eu » (Moussa, conversation, 6 avril 2009, Yeoville).

Ces projets de départ dépendent de la conjonction de plusieurs possibilités et contraintes. Ainsi, Malik qui a été refoulé lors d'une première tentative de partir en Europe et a passé un mois dans un centre de rétention au Maroc, poursuit simultanément plusieurs pistes pour avoir une deuxième chance. Il est en contact avec une Hollandaise "noire » qu'il a connue sur internet et dont il espère qu'elle peut le faire venir ; il demande aux personnes qui ont des contacts en Europe de «trouver des lettres d'invitation" de "n'importe quel pays en Europe » et se dit prêt à payer entre 200 et $300 \$$ pour une telle lettre (au total, il a réuni un budget de $4000 \$$ pour son départ en Europe); il a l'intention de faire une demande de visa auprès des ambassades polonaises ou grecques grâce auxquelles un ami a récemment réussi à se rendre en Europe. Aucune de ces possibilités n'aboutit. À cela s'ajoute l'expiration récente de sa carte de demandeur d'asile ce qui explique la montée de la pression pour changer son 
statut ou partir ailleurs. Un mois plus tard, il m'appelle de Bamako - il était en route pour l'Espagne.

\section{« Lui, il est différent des autres ! Il est sympa ! »}

Comme mentionné plus haut, les membres de la Tijaniyya viennent des différentes régions du Sénégal et se sont connus en Afrique du Sud grâce à leur réseau religieux. Comme au Sénégal et dans la plupart des pays de destination des migrants, ils s'organisent en dahiras Chaque dahira organise des rassemblements hebdomadaires et, lors de fêtes religieuses importantes, leurs membres se réunissent pour des manifestations nationales. Les dahiras sont des lieux de prière, d'instruction religieuse, de sociabilité et de soutien aux nouveaux venus auxquels elles fournissent souvent un logement, un emploi ou des microcrédits pour faire démarrer leurs affaires en Afrique du Sud.

Concernant leurs objectifs, les membres de la Tijaniyya mettent - beaucoup plus que les Maliens - l'accent sur le fait que la migration leur a permis d'apprendre et de faire des expériences. Quant à leurs perceptions et les relations à leur environnement social et spatial, comme les Maliens, ils disent avoir des «soucis de sécurité ». Mais le sujet apparaît moins fréquemment dans leurs récits, ils considèrent "qu'on essaie de se débrouiller avec ça » (10 octobre 2009, Troyeville) et ils n'associent pas la criminalité au racisme. Plutôt, ils voient la question en termes d'un "manque de confiance", "d'entraide » et "d'ouverture à l'autre ». Néanmoins, comme les Maliens, ils ne se sentent pas à l'aise quand ils se déplacent en ville. «Par exemple, si je commence par ici, il y a pas de liberté totale. Par exemple, il y a des heures, où tu dois pas sortir. Tu vois? Par exemple, ici, vers $19 \mathrm{~h}$, tout le monde est dans la maison. Et aussi, les gens ne sont pas ouverts trop. Ils se font pas confiance. Par exemple, si tu marches dans la rue, tu fais attention à l'autre [il rentre la tête dans les épaules] [...] S'il te voit, il pense que toi, tu vas l'agresser, ou tu vas le brutaliser, [...] tu vois, tu fais attention à lui et lui aussi, il fait attention à toi » (Mamadou, entretien, 7 mai 2010, CBD).

Leurs interactions avec les Sud-Africains les conduisent parfois aux mêmes stéréotypes que l'on trouve chez les Maliens. Un migrant qui emploie des Sud-Africains dans son magasin considère par exemple qu'ils ne sont "pas éduqués", que ce sont des «malades mentaux qui ne pensent qu'à faire la fête, aux filles et à l'alcool», qui "veulent la vie facile », qui ne sont pas « disciplinés » et qui « ne savent pas faire des économies " (Yéro, conversation, Troyeville, 10 octobre 2009). En même temps, selon un mécanisme bien connu, ces stéréotypes sont contredits par les interactions concrètes avec les Sud-Africains. Ainsi, lors de l'entretien cité plus haut, peu avant que Mamadou ne commence son récit sur le manque de confiance entre les gens en Afrique du Sud, un ami - sud-africain - qu'il a connu dans la boutique qu'il tient, passe pour lui faire une course ; après le départ de son ami, Mamadou affirme que « lui, il est différent des autres! Il est sympa !» (entretien, 7 mai 2010, СвD).

Comme les Maliens, les membres de la Tijaniyya affirment ne pas bouger beaucoup en ville à part pour se rendre au travail. Mais, certains d'entre eux possèdent une voiture ce qui leur permet de se déplacer plus facilement et leurs contacts sociaux, plus diversifiés, les conduisent à élargir leur périmètre dans la ville. Par rapport à leurs projets migratoires, si leur situation économique et administrative le permet, ils font 
des allers-retours entre le Sénégal et l'Afrique du Sud et visent à rentrer au Sénégal une fois qu'ils auront gagné assez d'argent pour réaliser leurs projets au Sénégal.

\section{" [Ce n'est] ni lié à la race, ni à la religion, ni à la nationalité. »} réfèrent souvent à "l'aventure $»^{21}$, ce qui englobe le choix de voyager pour gagner de l'argent pour la famille, les difficultés de la migration et, dans une moindre mesure, le fait de "vivre et de voir des choses". "Bon, nous Sénégalais, on est toujours à l'aventure quoi, grâce au sous-développement de notre pays quoi. Bon. C'est pas du tout facile, tu es seul, pas de famille, pas d'amis [...] Bon l'aventure c'est pas du tout facile quoi. C'est pas du tout facile. On a assez de problèmes aussi. Problèmes de sécurité. Problème de nostalgie de la famille. Problèmes de papiers " (Aziz, entretien, 4 août 2009, Rosebank). En même temps, ils soulignent que ces difficultés font "partie de la vie quoi pour nous » et qu'ils sont " prêt[s] à affronter toutes les conséquences » (Aziz, entretien, 4 août 2009, Rosebank).

À travers les mariages mixtes et les rassemblements religieux qui, contrairement à ce qui a cours chez les membres de la Tijaniyya, ne se limitent pas seulement aux ressortissants sénégalais, mais incluent des personnes venant d'autres pays africains ainsi que des Sud-Africains d'origine indienne, la question des interactions avec d'autres groupes et, plus largement, celle de la diversité sociale de la société sudafricaine est regardée dans une perspective très différente de celle des Maliens. Prenons l'exemple d'un migrant arrivé douze ans auparavant en Afrique du Sud et qui est "père de deux familles ", l'une au Sénégal et l'autre en Afrique du Sud. Pour lui, " réunir les deux familles » et faire en sorte qu'elles "s'adoptent ", notamment lors des visites de sa famille sud-africaine au Sénégal, représente " une mission impossible ». La difficulté réside dans les différences des deux cultures, "qui s'opposent comme le climat s'oppose", ce qui renvoie notamment à des problèmes de langue et de communication, mais aussi à des "traditions ", des "croyances ", des "manières de faire les choses", des "manières de voir la vie », de "s'habiller ", de "manger », de « communiquer » (Oumar, entretien, 4 août 2009, Rosebank).

Sur le thème de la diversité et de la mixité, il est intéressant de noter qu'on retrouve ici moins fréquemment les stéréotypes habituels des migrants envers les Sud-Africains. Aziz, en parlant des Sud-africains, mentionne «qu'ils sont moins africains que nous quoi », ce qui signifie qu'ils sont "moins attentieux [attentifs] aux cultures contrairement à nous " et ce qui renvoie aux différences dans les "cultures", les «traditions », et les «façons de communiquer ». Contrairement aux Maliens pour qui la diversité est une source de criminalité et de racisme, les Mourides la voient plutôt dans la perspective d'un long processus qui demande du travail, mais au terme duquel l'échange des deux cultures peut se réaliser. Ainsi, juste après avoir mentionné que réunir ses deux familles est une « mission impossible », Oumar constate : « mais c'est du travail à faire bon. Voilà quoi. Et puis [...] c'est un processus quoi qui va de longs temps quoi [...] Les cultures, pour l'une adopter à l'autre, bon, c'est du travail quoi, c'est pas du tout facile quoi, voilà ». Cependant, malgré ces difficultés, il "pense qu'avec les générations à venir [...] il y aura un échange d'idées qui est réel qui va pas se faire avec les femmes, mais avec les enfants qui vont grandir » (Oumar, entretien, 4 août 2009, Rosebank). 

aboutir à un entendement mutuel concerne non seulement le niveau personnel des mariages, mais se retrouve également, dans une sorte de structure de représentation(s) parallèle, au niveau de la société entière. Les migrants de ce groupe considèrent que l'Afrique du Sud est un pays « qui vient de s'ouvrir ». Certaines personnes en Afrique du Sud ne "savaient pas ce que c'était un aventurier » et cela a amené une "différence " et un «manque d'entente ». Et, ici encore, « ça demande un temps quoi, c'est du temps pour s'améliorer, s'adapter aux gens quoi ». « En Afrique, nous aussi on connaît bien que c'est un pays qui vient d'ouvrir quoi, ça va pas être facile, mais au fur et à mesure qu'on se mixe il y aura une amélioration quoi, réelle des trucs quoi » (Aziz, entretien, 4 août 2009, Rosebank).

À partir de ce thème, Aziz revient sur la question de la xénophobie. Contrairement aux Maliens, il ne fait pas d'amalgame entre criminalité, diversité et racisme. Pour lui, la xénophobie n'est pas liée à la criminalité et, plus important encore, elle est dissociée des «différences» et du «manque d'entente» dont il vient de parler. Pour lui, la xénophobie est assez distante, limitée "aux ghettos comme Alexandra». Il la voit comme des actions d'une «bande de faiseurs de mauvais ", de "gens qui n'ont pas de travail » et qui veulent faire la « révolution » pour se «faire entendre ». Pour lui, en raison de ses échanges avec les Sud-Africains, la "xénophobie » n'est pas une " réalité » et, surtout, il soutient que «moi, je vois pas la xénophobie intérieurement aux gens quoi ». "Même si on a pas de femmes mariées, ils ont des filles pour sortir avec, pendant des dizaines d'années depuis 1994, c'est vrai depuis 1997, donc surtout avec une fille et puis vivre ensemble. Bon, si c'était la xénophobie, bon, et puis non seulement dans les bars, partout il y a des amis sud-africains, différentes nationalités, et puis on se lance dans le même mouvement et puis voilà quoi. Bon, sur nous, vers les Sénégalais, d'après mon propre, il y a pas quelque chose comme ça quoi. Ça se parle un peu partout dans la radio, des trucs, mais moi, j'ai vécu ici maintenant depuis 1997, mais j'ai pas vécu ça comme une réalité quoi » (Aziz, entretien, 4 août 2009, Rosebank).

Pour soutenir ses propos, Aziz parle ensuite des endroits où un échange entre migrants et Sud-Africains et, plus généralement, entre personnes de nationalités différentes se met en place. Il s'agit notamment du travail, un marché touristique, où les vendeurs africains s'entraident au point qu' "il n'y a pas de frontières ", et des rassemblements religieux telles les cérémonies pour l'enterrement d'un imam qui réunissent différentes nationalités africaines et «même des Indiens d'ici ». Il en conclut qu'« on se lie, soit par le travail, soit par la religion » et mentionne également des activités de loisirs ; s'il va dans un bar, « on laisse la religion à la porte. On danse, on discute, on échange ».

Ces migrants soulignent qu'il s'agit d'un échange réel, qu'ils sont "prêts à se lier ", qu'on «s'adopte» et "s'accepte", qu'on «se lie par ce qu'on a en commun », et ils mettent l'accent sur le fait de " communiquer ", de se « connecter ", de se "découvrir " et «d'aller vers des positivités" sans être «ni lié à la race, ni à la religion, ni à la nationalité ». Dans cette perspective, ils soulignent qu'ils «ne jugent pas l'homme » sur les apparences comme la couleur de la peau ou de l'habillement, mais par son « comportement » et par «ce qu'il a dans la tête» (Amadou, entretien, 23 mai 2010, marché touristique près de Johannesburg).

En conséquence, ces migrants dont les familles sont présentes en Afrique du Sud, qui se trouvent dans une situation économique et administrative stable et qui disposent de ressources sociales et spatiales plus importantes que les Maliens, ne pensent que 
rarement à partir ailleurs. Tout comme les membres de la Tijaniyya, ils considèrent que «l'Europe est saturée maintenant " (Yero, conversation, 10 octobre 2009, Troyeville) et soulignent l'avantage de l'Afrique du Sud par rapport aux pays européens : il est plus facile d'y ouvrir un commerce ou un compte bancaire, d'y acheter une maison, et de s'y marier et, contrairement à la France où les gens s'entassaient dans les foyers, les conditions de vie y étaient meilleures (Moustapha, entretien le 12 novembre 2008, Rosebank). Ici apparaît la deuxième face de la notion de people as infrastructure de Simone. Certes, "life in the inner city fosters intense cooperation among fellow nationals and ethnics", mais en même temps elle est faite "d'interactions inattendues" et de " conjonctures d'activités hétérogènes" "[which] can be used to rehearse new ways of navigating complex urban relationships and to construct a sense of commonality that goes beyond parochial identities » (Simone 2008: 71, 75 et 88).

\section{Repli sur soi et ouverture vers l'autre}

37 Ainsi, les représentation(s) de la migration et de la réussite et les modes d'insertion dans la ville des migrants sénégalais et maliens à Johannesburg, notamment le regard sur la relation entre objectifs économiques et éducatifs de la migration, sur leur environnement social et les manières de s'y lier et, en fin de compte, sur leurs projets d'avenir, varient considérablement d'un groupe à l'autre, d'un environnement social à l'autre, d'un quartier à l'autre. Alors que les Maliens valorisent surtout les bénéfices monétaires, les membres de la Tijaniyya soulignent les expériences qu'ils ont pu faire en voyageant et les Mourides tiennent un discours classique sur les difficultés du voyage et les efforts pour les surmonter. Les hauts objectifs en termes de réussite des Maliens contrastent avec leurs situations professionnelles parfois instables et avec une perception très négative de leur environnement social. Dans leurs récits, les problèmes de sécurité et la criminalité auxquels ils se voient confrontés sont directement liés au racisme et à la discrimination sur le marché de l'emploi dont ils se considèrent les victimes. Ceci est alimenté par un nombre d'incidents plus élevés que dans les autres groupes. Pour faire face à cette situation, ils limitent leurs déplacements sur des espaces très réduits et leurs relations sociales à des personnes de confiance, notamment au sein des réseaux familiaux dont ils font partie.

En revanche, les termes de racisme, de criminalité, et de xénophobie n'apparaissent que rarement dans les récits des membres de la Tijaniyya et de la confrérie mouride, qui décrivent la situation en termes d'un "manque de confiance", "d'ouverture à l'autre », de "problèmes d'entente » et de "communication». Les Mourides voient la question de l'adaptation et de l'intégration en Afrique du Sud comme un travail de longue haleine qui aboutira - et aboutit déjà à travers les familles mixtes, les échanges professionnels et au sein des mouvements religieux - à un "échange " mutuel et à des " positivités " n'étant «ni lié à la race, ni à la religion, ni à la nationalité ». En même temps, les problèmes de xénophobie sont assez éloignés des préoccupations des Mourides. Ces perceptions sont également liées au fait que la plupart des Mourides, et ceci est valable dans une certaine mesure pour les membres de la Tijaniyya, après parfois quinze ans en Afrique du Sud, ont acquis des situations économiques confortables qui leur ont permis de sortir des quartiers où les incidents de sécurité sont plus fréquents et de s'installer dans des quartiers plus aisés. Grâce aux contacts qu'ils 
ont pu nouer au travail ou dans les mouvements religieux, ils disposent d'une maitrise d'espaces plus diversifiés que les Maliens.

Premièrement, ces situations diverses s'expliquent par la diversité sociale des réseaux sociaux dans lesquels les migrants sont insérés. Pour les Maliens, la famille (élargie), dont les membres ont tous des profils socioéconomiques très semblables, constitue la principale ressource de contacts sociaux. Pour eux, il existe un lien causal entre criminalité, racisme et la diversité sociale de la société sud-africaine. En revanche, ceux qui interagissent plus avec des Sud-Africains et d'autres groupes de migrants développent une vision plus positive de cette diversité. Ces interactions les exposent à des environnements sociaux divers qui leur permettent de changer de perspective et considérer leurs représentation(s) à partir d'un autre "point de vue " (Blickpunkt) (Cassirer 1999b : 24) ; les différentes interprétations et explications de la diversité de la société sud-africaine en est l'exemple le plus saillant. Pour eux, l'entente sociale est un travail de longue haleine, mais elle est possible. Bien qu'il existe des stéréotypes, ceuxci se voient parfois contredits par des relations d'entraide mutuelle et des amitiés entre migrants et Sud-Africains. Mais on voit également des différences dans les situations économiques des migrants qui vont de travailleurs temporaires, en particulier dans le commerce, à des commerçants établis qui gèrent leurs propres magasins de vente au détail ou en gros ou des cybercafés. Ceci renvoie également à l'importance des réseaux sociaux et aux ressources qu'ils offrent; les Maliens soutiennent les nouveaux venus, mais en raison de leur nombre limité, ils sont beaucoup moins en mesure de leur fournir un premier emploi que les réseaux plus larges et divers de la Tijaniyya et des Mourides.

La question des parcours professionnels renvoie à une deuxième raison qui influe sur les perceptions et les stratégies des migrants. Les différents groupes se trouvent aussi à des étapes différentes du parcours migratoire. Parmi les Maliens, on trouve un grand nombre de jeunes célibataires qui viennent d'arriver et sont soumis à la pression sociale de gagner de l'argent, de se marier, d'envoyer de l'argent à la famille - ou de se rendre dans des endroits aussi prestigieux que l'Europe. La plupart des autres migrants sont plus âgés et/ou professionnellement plus établis. Ils sont propriétaires de magasins, de boutiques ou de cybercafés, mariés au pays ou en Afrique du Sud, ou ont fait venir leur femme et leurs enfants. Ces positions différentes dans le cycle migratoire font aussi l'objet de mises en récit différentes. Tandis que les discours des Maliens sont parfois pleins d'incertitudes, de désespoir, mais aussi d'espoirs et d'attentes concernant leur futur, les membres de la Tijaniyya balancent entre avantages et désavantages en Afrique du Sud pour en conclure qu'il faut accepter les derniers pour profiter des premiers. Les récits des Mourides aboutissent à une véritable cohérence du vécu. Ils parlent de la nécessité de venir en Afrique du Sud pour gagner de l'argent et soutenir la famille au Sénégal et des souffrances qu'ils ont dû supporter comme la recherche du travail, des conditions de vie difficiles, des solutions à la question des papiers. Ils parlent des difficultés qu'ils ont dû surmonter, notamment la criminalité ou le danger de « déraper " dans un contexte ou " tout est à la portée ", notamment "les femmes " et « l'alcool ", et déclarent que c'est grâce à une « conscience forte ", à une " éducation solide ", aux " valeurs ", à la religion et au soutien des mouvements religieux qu'ils ont pu surmonter ces difficultés pour finalement réussir (Amadou, entretien, 23 mai 2010, marché touristique près de Johannesburg). La mise en récit rétrospective de ces expériences migratoires fait penser à la coexistence d'éléments contradictoires chez Mannoni (1969) ou la «narration de soi » chez Paul Ricœur. Ricœur établit une liaison 
étroite entre temps et narration dans la visée d'une construction de représentation(s) complexes et réflexives du temps vécu; il n'est «temps pensé que raconté » (Ricœur 1985 : 435). Le récit possède ainsi la capacité d'intégrer des ruptures événementielles dans une continuité temporelle et spatiale à travers une structure que Ricœur appelle « concordance-discordance ». La dynamique de «mise en intrigue » de faits permet la stabilité de l'histoire racontée, qui provient de la capacité d'intégrer des ruptures et des changements, et rejoint les considérations de Ricœur sur l'identité (1990).

Cette deuxième interprétation en termes de position dans le parcours migratoire complète ainsi la première, qui insiste sur l'importance des réseaux sociaux et notamment sur leur diversité sociale pour les transformations des représentation(s) des migrants. L'importance des réseaux sociaux provient surtout d'un contexte où le groupe de migrants est relativement petit et où l'environnement social est perçu comme hostile. Ces migrants se voient davantage contraints à avoir - concrètement et psychologiquement - recours aux réseaux que des migrants en France par exemple. En conséquence, on assiste à peu de mises en questions des hiérarchies sociales et des processus d'individuation dont mes recherches antérieures ont montré qu'elles jouent un rôle important dans la transformation des représentation(s) de la migration et de la réussite chez des migrants originaires de la vallée du fleuve Sénégal en France (Ludl 2008a).

Cette importance des réseaux sociaux sénégalais et maliens à Johannesburg constitue une spécificité. En effet, contrairement à d'autres groupes de migrants à Johannesburg, il existe très peu de divisions et conflits internes au sein de la communauté sénégalaise et malienne (Simone 2008: 77) ${ }^{22}$. On l'a vu, certains des Sénégalais et Maliens sont « battered by the demands of maintaining the semblance of a safe domestic environment, [and] find few incentives to exceed the bound of personal survival» ce qui mène à une "intricate territorialization and a patchwork of zones of relative security " (Simone $2008: 75$ ). En même temps, on constate « the capacity of individual actors to circulate across and become familiar with a broad range of spatial, residential, economic, and transactional positions [...] and an implicit willingness to interact with one another in ways that draw on multiple social positions " (Simone 2008: 75). En revanche, là où Simone identifie un affaiblissement des références nationales et «ethniques », ces deux cas de figure dont il parle, un repli sur soi et une ouverture vers l'autre, se jouent dans le contexte d'une forte stabilité et fonctionnalité des réseaux sociaux. En conséquence, les "interactions inattendues", les « interdépendances émergentes» avec des « antagonistes réels et potentiels » et la « conjoncture d'activités hétérogènes" qui se trouvent au cœur de la notion de people as infrastructure résultent moins de la densité de l'espace et de la seule présence d'Africains d'origines nationales et "ethniques " très diverses dans le centre-ville de Johannesburg que de réseaux sociaux qui s'appuient sur des relations de confiance liant des personnes à travers des espaces aussi larges et divers que Rosebank, Fordsburg, ou le CBD à Johannesburg, Dakar, ou Nairobi.

Pour conclure, je voudrais insister sur l'imbrication entre relations sociales, représentation(s) et relation à l'espace. D'un côté, comme on l'a vu, les premières jouent un rôle de moteur pour les transformations des représentation(s) des migrants. De l'autre, les relations sociales s'avèrent également cruciales pour la relation à l'espace. Ce sont des «social relations stretched over space» (Massey 1994: 14) qui se trouvent au cœur d'un processus de fabrication de lieux (placemaking). Les endroits et les quartiers que les migrants fréquentent et avec lesquels ils sont familiers dépendent 
largement de qui ils connaissent, à qui ils vont rendre visite, et avec qui ils vont se déplacer. Des endroits spécifiques comme ceux des manifestations religieuses ou la boutique des Maliens deviennent des «lieux " parce qu'ils sont des lieux de sociabilité où les migrants se sentent en sécurité et parce qu'ils les associent à des significations spécifiques. Comme l'un des membres de la Tijaniyya l'exprime après leur rassemblement hebdomadaire, "c'est le travail et ici, donc c'est très important " (10 octobre 2009, Troyeville). Dans cette perspective, les déplacements dans la rue comme Mamadou les décrit, font également l'objet d'une fabrication des lieux; si les piétons «font attention » les uns aux autres, même si c'est par prudence et méfiance, des relations sociales, bien qu'anonymes et éphémères, se nouent dans la rue. À cela s'ajoute que cette définition de la notion de lieux inclut non seulement les relations sociales locales, mais également celles qui vont au-delà des espaces locaux. "Places, in fact, are always constructed out of articulations of social relations [...] which are not only internal to that locale but which link them to elsewhere » (Massey 1995 : 183). Ces relations, au-delà des espaces locaux, s'avèrent particulièrement importantes dans un contexte migratoire. En effet, la plupart des migrants sont en contact avec leurs familles restées dans leur pays d'origine et avec des amis et de la famille habitant dans d'autres pays. Dans ce cas-là, il s'agit de pays en Afrique, en Europe et en Asie - des lieux que les migrants ont visités ou ne connaissent pas encore et qui sont, grâce aux relations sociales, « fabriqués » entre réalité et imaginaire, constituant de véritables « espaces de possibilités» (Schiffauer 2006) et plaçant l'imaginaire au cœur de la "spatial and temporal openness of the city» (Mbembe et Nuttall $2004: 361$ ).

\section{BIBLIOGRAPHIE}

ADAMS, A. 1977. Le long voyage des gens du fleuve. Paris, Maspero.

ADELKHAH, F. et J.-F. BAYART. 2007. «Introduction. Pour une anthropologie politique du voyage », in F. ADELKHAH, J.-F. BAYART (éd.) Voyages du développement. Paris, Karthala : 5-29.

BÂ, Ch. O. 1996. Dynamiques migratoires et changements sociaux au sein des relations de genre et des rapports jeunes/vieux des originaires de la moyenne vallée du fleuve Sénégal, Thèse de doctorat de troisième cycle d'anthropologie, Dakar, Faculté des lettres et sciences humaines, département anthropologie, Université Cheikh Anta Diop.

BALLO, M. 2009. Migration au Mali. Profil national 2009. Genève, Organisation internationale pour les migrations, $136 \mathrm{p}$.

BAROU, J. 2001. « Les logiques modernes de l'immigration, entretien avec J. BAROU », propos recueillis par Olivier BARLET, Grenoble, octobre 2001, publié le 21 octobre 2002, disponible sur : http://www.africultures.com/index.asp?menu=affiche_article\&no=63, [consulté le 20 mai 2006].

BAVA, S. 2000. « Reconversions et nouveaux mondes commerciaux des mourides à Marseille», Hommes \& Migrations, $1224:$ 46-55. 
BAVA, S. 2002. «Entre Marseille et Touba : le mouride migrant et la société locale », in M. C. DIOP (éd.), La société sénégalaise entre le local et le global. Paris, Karthala : 579-596.

BAVA, S. et C. GUEYE. 2001. «Le grand magal de Touba : exil prophétique, migration et pèlerinage au sein du mouridisme ", Social Compass, 48(3) : 421-438.

BAYART, J.-F. 1996. L'illusion identitaire. Paris, Fayard.

BAYART, J.-F. 2004. Le gouvernement du monde. Une critique de la globalisation. Paris, Fayard.

BAYART, J.-F. 2006. L'État en Afrique. La politique du ventre. Nouvelle édition, Paris, Fayard.

BAYART, J.-F. et J.-P. WARNIER (éd.). 2004. Matière à politique. Le pouvoir, les corps et les choses. Paris, Karthala.

BEINART, W. 1992. « Political and collective violence in Southern African historiography », Journal of Southern African Studies, 18(3) : 455-486.

BEKKER, S., I. EIGELAAR-MEETS, E. GARY et al. 2008. Xenophobia and Violence in South Africa. A Desktop Study of the Trends and a Scan of Explanations Offered. Stellenbosch, Stellenbosch University, rapport non publié.

BOUILLON, A. 1999. L'immigration africaine en Afrique du Sud. Les migrants francophones des années 1990. Paris, Karthala.

BREDELOUP, S. et O. Pliez. 2005. « Migrations entre les deux rives du Sahara. Éditorial », Autrepart, $4(36): 3-20$.

BREMNER, L. et P. SUBIRÓs. 2007. Johannesburg : Emerging/Diverging Metropolis. Mendrisio (CH), Mendrisio Academy Press.

BUGGENHAGEN, B. A. 2002. «Prophets \& profits : Gendered \& generational visions of wealth \& value in Senegalese Murid households », Journal of Religion in Africa, XXXI(4) :373-401.

BUGgenhagen, B. A. 2004. « Domestic object(ion)s : The Senegalese Murid trade diaspora and the politics of marriage payments, love, and state privatization », in B. WEISs (éd.), Producing African Futures. Ritual and Reproduction in a Neoliberal Age. Leyde, Brill Academic Press : 21-53.

CARTER, D. M. 1997. States of Grace. Senegalese in Italy and the New European Immigration. Minneapolis, University of Minnesota Press.

CASSIRER, E. [1937] 1999a. « Die Invarianten der Wahrnehmung und des Begriffs », in E. CASSIRER et al. (eds.), Ziele und Wege der Wirklichkeitserkenntnis. Nachgelassene Manuskripte und Texte, vol. 2. Hambourg, Meiner : 83-133.

CASSIRER, E. 1999b. Ziele und Wege der Wirklichkeitserkenntnis. Nachgelassene Manuskripte und Texte, vol. 2, J. M. KROIS et K. C. KÖHNKE (éd.). Hambourg, Meiner.

CASSIRER, E. [1929] 2002. Philosophie der symbolischen Formen, 3. Phänomenologie der Erkenntnis, in E. CASSIRER, Gesammelte Werke, Hamburger Ausgabe, vol. 13, J. CLEMENS et B. RECKI (éd.). Hambourg, Meiner.

CASTORIADIS, C. 1999. L'institution imaginaire de la société. Paris, Seuil (Essais/points).

COPANS, J. 1989. Les marabouts de l'arachide. La confrérie mouride et les paysans du Sénégal. Paris, L'Harmattan. 
CORMSA (Consortium for Refugees and Migrants in South Africa). 2008. Protecting Refugees and Asylum Seekers, and Immigrants in South Africa. 73 p., disponible sur : http://www.cormsa.org.za/ wp-content/uploads/2008/06/cormsa08-final.pdf, [consulté le 20 octobre 2008].

Coulon, C. 1981. Le Marabout et le prince : islam et pouvoir au Sénégal. Paris, Pedonne.

COULON, C., O. CRUISE et B. DONAL. 1988. Charisma and Brotherhood in African Islam. Oxford, Clarendon Press.

CRUISE, O. et B. DONALD. 1975. Saints and Politicians. Essays in the Organisation of a Senegalese Peasant Society. Londres, Cambridge University Press.

CRUSH, J., D. MC DONALD, V. WILliams et al. 2008. The Perfect Storm. The Realities of Xenophobia in Contemporary South African Discourses on Immigration: Changes and Continuity in the Transition Period. Cape Town, Idasa and Kingston, Ontario, Southern African Research Centre, Southern African Migration Project (Migration Policy Series ; 50), 67 p.

DAUM, C. 1998. Les associations de Maliens en France. Migration, développement et citoyenneté. Paris, Karthala.

DELEUZE, G. 1990. Pourparlers, 1972-1990. Paris, Minuit.

DENIAU, X. 1996. Migration et développement au Mali. Rapport d'information sur la mission effectuée par une délégation de la Commission au Mali. Paris, Assemblée nationale, Commission des affaires étrangères.

DIOUF, M. 2000. « The Senegalese Murid trade diaspora and the making of a vernacular cosmopolitanism », Public Culture, 12(3) : 679-702.

DONEGANI, J.-M., S. DUCHESNE et F. HAEGEL. 2002. «Sur l'interprétation des entretiens de recherche », in J.-M. DONEGANI, S. DUCHESNE et F. HAEGEL (éd.), Aux Frontières des attitudes entre le politique et le religieux. Textes en hommage à Guy Michelat. Paris, L'Harmattan : 273-295.

DUCHESNE, S. 1996. « Entretien non préstructuré, stratégie de recherche et étude des représentations. Ou : Peut-on déjà faire l'économie de l'entretien "non-directif" en sociologie?» Politix, 35 : 189-206.

DUCHESNE, S. 2000. «Pratique de l'entretien non-directif », in M. BACHIR (éd.), Les méthodes au concret. Démarches, formes de l'expérience et terrains d'investigation en science politique. Paris, PUF : 9-30.

DUCHESNE, S. et F. HAEGEL. 2005. L'enquête et ses méthodes. L'entretien collectif. Paris, Armand Colin.

FALL, P. D. 2003. Migration internationale et droits des travailleurs au Sénégal, rapport dans le cadre du REMIGRAF-IFAN (Réseau d'étude des migrations internationales africaines), Université Cheikh Anta Diop de Dakar, 49 p., disponible sur : http://portal.unesco.org/shs/en/file_download.php/ 3af480525acd8aaa26af490bd72d4a1cRapport+Senegal+Demba+Fall.pdf, [consulté le 14 février 2005].

FALL, P. D. 2004. « Les Sénégalais au Kwazulu-Natal (Afrique du Sud) ou les "naufragés" de la migration internationale ", communication présentée au Congrès international d'études africaines, Barcelone, Espagne, 12-15 janvier 2004, 10 p.

FAYE, I. L., 2008. « Émigrés sénégalais en Afrique du Sud. La "Teranga” à Grahamstown », SUD Quotidien, 22 septembre.

FAYE, O. 2001. De «l'économie réelle » au marché Sandaga (Dakar) : les pratiques des talibés Mourides dans les transactions, Dakar, CODESRIA. 
FERON, E. et M. HASTINGS. 2002. «Introduction », in E. FERON et M. HASTINGS (éd.) L'imaginaire des conflits communautaires. Paris, L'Harmattan : 11-22.

GEERTZ, C. 1993a. «Ethos, world view, and the analysis of sacred symbols ", in C. Geertz (ed.), The Interpretation of Cultures. Hammersmith, Londres, Fontana Press : 126-141.

GEERTZ, C. 1993b. « Religion as a cultural system », in C. GEERTZ (ed.), The Interpretation of Cultures. Hammersmith, Londres, Fontana Press : 87-125.

GILROY, P. 2000. Against Race. Imagining Political Culture beyond the Color Line. Cambridge, Belknap Press of Havard Unversity Press.

GONIN, P. 2005. « Jeux d'acteurs et enjeux territoriaux : quelles migrations pour quel développement ? L'exemple du bassin du fleuve Sénégal (République du Mali) », in M. CHAREF, P. GONIN (éd.), Émigrés - Immigrés dans le développement local. Agadir, ORMES/Sud Contact : 259-278.

HAVARD, J.-Fr. 2001. «Ethos “bul faale” et nouvelles figures de la réussite au Sénégal », Politique africaine, (82) : 63-77.

HSRC. 2008. Citizenship, Violence and Xenophobia in South Africa. Perceptions from South African Communities. Pretoria, Human Sciences Research Council, disponible sur http://www.hsrc.ac.za/ Document-2807.phtml, [consulté le 23 novembre 2010], 59 p.

IDASA. 2008. «Aliens, migrants, refugees and interlopers. Perceptions of foreigners in South Africa », ePoliticsSA (1)

LANDAU, L. B. 2010. « Loving the alien? Citizenship, law, and the future in South Africa's demonic society », African Affairs, 109(435) : 213-230.

LANDAU, L. B. et I. FREEMANTLE. 2010. « Tactical cosmopolitanism and idioms of belonging : insertion and self-exclusion in Johannesburg », Journal of Ethnic and Migration Studies, 36(3) : 375-390.

LANDAU, L. B. et V. GINDREY. 2008. « Migration and population trends in Gauteng Province 1996-2055 ", Migration Studies Working Paper Series, \# 42, Johannesburg, Forced Migration Studies Programme, University of the Witwatersrand, $31 \mathrm{p}$.

LEKOGO, R. 2006. « Francophone Africans in Cape Town: A failed migration? », in C. CRoss et al. (eds.) View on Migration in sub-saharan in Africa. Proceedings of an African Migration Alliance Workshop. Cape Town, South Africa, HSRC Press : 207-219.

LIMA, S. 2003. Découpage entre espace et territoire : la fin des limites? La fabrique des territoires communaux dans la région de Kayes, Mali. Thèse de doctorat de géographie, Poitiers, Université de Poitiers.

LLITERAS, S. M. 2009. « A path to integration: Senegalese Tijanis in Cape Town », African Studies, $68(2): 215-233$.

LUDL, C. 2008a. La (les) Représentation(s) de la migration, entre pouvoir et réussite. La mobilité des migrant(e)s originaires de la Vallée du fleuve Sénégal entre leurs pays d'origine et la France. Thèse de doctorat, Paris, Institut d'études politiques de Paris, Berlin, Otto-Suhr-Institut für Politikwissenschaft der Freien Universität Berlin.

LUDL, C. 2008b. " "To skip a step" : New representation(s) of migration, success and politics in Senegalese rap and theatre ", Stichproben. Wiener Zeitschrift für kritische Afrikastudien, 14 : 97-122. Disponible sur : http://www.univie.ac.at/ecco/stichproben/Nr14_Ludl.pdf.

LUDL, C. À paraître. "Ambivalent Cosmopolitans" ? Representations of Mobility and Success of Senegalese and Malian Migrants in Johannesburg, communication présentée à la master class 2010 « Clash of 
Cultures? », université de Constance, Allemagne, 19-27 juillet, à paraître in A. WA KABWE-SEGATTI (ed.), Rethinking Migration in Contemporary Southern Africa: Methods, Knowledge Circulation and Subjectivities.

MANCHUELLE, F. 1997. Willing Migrants : Soninke Labor Diasporas, 1848-1960. Athens, Ohio, Ohio University Press.

MANNONI, O. 1969. «Je sais bien, mais quand même », in O. Mannoni (éd.), Clefs pour l'imaginaire ou L'autre scène. Paris, Seuil : 7-33.

MARTIN, D.-C. 1999. Coon Carnival, New Year in Cape Town: Past to Present, Le Cap, David Philip.

MARTIN, D.-C. 2008. « Can jazz be rid of the racial imagination? Creolization, racial discourses, and semiology of music », Black Music Journal, 28(2) : 105-123.

MARTIN, D.-C. et R. EBRAHIM-VALLY. 2006. « Viewing the "New” South Africa. Representations of South Africa in television commercials: An experiment in non-directive methods ", Questions de Recherche / Research in Question, 19, 98 p, disponible sur : http://www.ceri-sciences-po.org/ publica/question/qdr19.pdf, [consulté le 27 février 2007].

MASSEY, D. 1994. Space, Place and Gender. Minneapolis, University of Minnesota Press.

MASSEY, D. 1995. « Places and their pasts », History Workshop Journal, 39 : 182-192.

MATTES, R., D. TAYLOR, D. MCDONALD et al. 1999. Still Waiting for the Barbarians: SA Attitudes to Immigrants and Immigration. Idasa and Kingston, Ontario, Cape Town, Southern African Migration Project (Migration Policy Series ; 14) : 1999, 39 p.

MBEMBE, A. et S. NUTTALl. 2004. «Writing the World from an African Metropolis », Public Culture, $16(3): 347-372$.

MICHELAT, G. 1975. « Sur l'utilisation de l'entretien non-directif en sociologie », Revue française de sociologie, $16: 229-247$.

MISAGO, J.-P., L. B. LANDAU et T. MONSON. 2009. Towards Tolerance, Law, and Dignity. Addressing Violence against Foreign Nationals in South Africa. Johannesburg, International Organisation for Migration (Іом).

MORRIS, A. 1994. « The desegregation of Hillbrow, Johannesburg, 1978-1982 », Urban Studies, 31(6) : 821-834.

MORRIS, A. 1999. Bleakness and Light. Inner City Transition in Hillbrow, Johannesburg. Johannesburg, South Africa, Witwatersrand University Press.

MURRAY, M. J. 2008. Taming the Disorderly City. The Spatial Landscape of Johannesburg after Apartheid. Ithaca, Cornell University Press.

N’DONGO, S. 1975. Le voyage forcé. Itinéraire d'un militant. Paris, Maspero.

NEOCOSMOS, M., From "Foreign Natives" to "Native Foreigners" - Explaining Xenophobia in Post-Apartheid South Africa : Citizenship and Nationalism, Identity and Politics. Dakar, Codesria.

PALMERY, I. 2002. Refugees, Safety and Xenophobia in South African Cities. The role of Local Government. Johannesburg, CsvR.

PIAN, A. 2009. « La fabrique des figures migratoires depuis l'expérience des migrants sénégalais », Journal des Anthropologues, 118-119 : 249-277. 
POLZER, T. et V. IGGLESDON. 2009. Humanitarian Assistance to Internally Displaced Persons in South Africa. Lessons Learned Following Attacks on Foreign Nationals in May 2008. Johannesburg, Forced Migration Studies Programme, University of the Witwatersrand, Report.

QUIMINAL, C. 1991. Gens d'ici, gens d'ailleurs. Paris, Christian Bourgois.

RICCIO, B. 2006. « "Transmigrants” mais pas "nomades”. Transnationalisme mouride en Italie », Cahiers d'études africaines, XLVI(1) : 95-114.

RICEUR, P. 1985. Temps et récit, III. Le temps raconté. Paris, Seuil.

RICœUR, P. 1990. Soi-même comme un autre. Paris, Seuil.

ROGERS, C. 1945." The non-directive method as a technique for social research », American Journal of Sociology, 50(4) : 279-283.

SAIRR. 2008. Statement by the South African Institute of Race Relations on Causal Factors behind the Violent Unrest in and around Johannesburg - 20th May 2008, South African Institute of Race Relations, disponible sur : http://www.sairr.org.za/press-office/archive/statement-by-thesouth-african-institute-of-race-relations-on-causal-factors-behind-the-violent-unrest-in-andaround-johannesburg-20-may-2008.html, [consulté le 23 octobre 2009].

SANDKÜHLER, H. J. 1999. « Repräsentation », in H. J. Sandkühler (ed.), Enzyklopädie Philosophie, 2 volumes. Hambourg, Meiner : 1384-1389.

SCHIFFAUER, W. 2006. « Transnationale Solidaritätsgruppen, imaginäre Räume, irreale Konditionalsätze », in H. BERKING (ed.), Die Macht des Lokalen in einer Welt ohne Grenzen. Frankfort/ New York, Campus Verlag : 165-180.

SCHMIDT DI FRIEDBERG, O. et R. BLION. 2000. « Du Sénégal à New York, quel avenir pour la confrérie mouride? ", Hommes \& Migrations, 1224 : 36-45.

SHARP, J. 2008a. «"Fortress SA" : Xenophobic violence in South Africa », Anthropology Today, 24(4) : $1-3$.

SHARP, J. 2008b.« "Fortress SA" ? John Sharp Responds », Anthropology Today, 24(5) : 22.

SIMONE, A. 2008. «People as infrastructure : Intersecting fragments in Johannesburg », in A. MBEMBE et S. NUTTALL (eds.), Johannesburg. The Elusive Metropolis. Johannesburg, Wits University Press : 68-90.

STREIFF-FÉNART, J. et P. POUTIGNAT. 2006. « De l'aventurier au commerçant transnational, trajectoires croisées et lieux intermédiaires à Nouadhibou (Mauritanie) », Cahiers de la Méditerranée, 73, disponible sur : http://cdlm.revues.org/index1553.html [consulté le 22 novembre 2010].

TALL, S. M. 1998. « Un instrument financier pour les commerçants et émigrés mourides de l'axe Dakar-New York : Kara International Exchange », in L. HARDING, L. MARFAING et M. sow (éd.), Les opérateurs économiques et l'État au Sénégal. Hambourg, Lit : 73-90.

TALL, S. M. 2002. «L'émigration internationale sénégalaise d'hier à demain », in M. C. DIOP (éd.), La société sénégalaise entre le local et le global. Paris, Karthala : 549-578.

TAYLOR, C. 2004. Modern Social Imaginaries. Durham, Londres, Duke University Press.

TIMERA, M. 1996. Les Soninké en France. D’une histoire à l'autre. Paris, Karthala.

TRIAUD, J.-L. et D. ROBINSON (éd.). 2000. La Tijaniyya : une confrérie musulmane à la conquête de l'Afrique. Paris, Karthala. 
TURINO, T. 2000. Nationalists, Cosmopolitans, and Popular Music in Zimbabwe. Chicago, University of Chicago Press.

TURNER, V. 1977. « Process, system, and symbol. A new anthropological synthesis », Dadalus, $1(106): 61-80$

TURNER, V. 1992. The Anthropology of Performance. New York, PAJ Publications.

VIGNESWARAN, D. et M. DUPONCHEL. 2009. One Burden Too Many? A Cost-Benefit Analysis of Immigration Policing in Gauteng. Johannesburg, The Forced Migration Studies Programme at the University of the Witwatersrand, Open Society Foundation for South Africa, $31 \mathrm{p}$.

VIGouroux, C. B. 2005. " "There are no Whites in Africa" : Territoriality, language, and identity among Francophone Africans in Cape Town », Language \& Communication, 25 : 237-255.

VIGouroux, C. B. 2008. " "The smuggling of La Francophonie" : Francophone Africans in Anglophone Cape Town (South Africa) ", Language in Society, 37(3) : 415-434.

WA KABWE-SEGATTI, A. 2008. «Violences xénophobes en Afrique du Sud : retour sur un désastre annoncé », Politique africaine, 112 : 99-118.

WA KABWE-SEGATTI, A. 2009. «"We offer the whole of Africa here!” African curio traders and the marketing of a global African image in post-apartheid South African Cities ", Cahiers d'études africaines, 193-194(1-2) : 285-308.

WA KABWE-SEGATTI, A. et C. FAUVELLE-AYMAR. 2009. «Institutions, political participation and violence », communication présentée au colloque Exorcising the Demon within: Xenophobia, Violence, and Statecraft in Contemporary South Africa, 22 mai 2009, University of the Witwatersrand, Johannesburg.

ZEKERIA OULD, A. S. 2001. «'Tcheb-tchib’ et compagnie. Lexique de la survie et figures de la réussite en Mauritanie », Politique africaine, $82: 78-100$.

\section{NOTES}

1. Cet article s'appuie sur des recherches menées dans le cadre du programme ANR Mitrans (Migrations de transit en Afrique. Dynamiques locales et globales, gestion politique et expériences d'acteurs) mené par l'Urmis-Soliss, Paris-Nice, l'Institut français d'Afrique du Sud, Johannesburg et le Forced Migration Studies Programme, University of the Witwatersrand, Johannesburg.

2. En 2007 , les migrants internes représentaient $37,4 \%$ des habitants de la province du Gauteng, les personnes nées à l'étranger en constituaient $5,6 \%$ et les trois quarts de la croissance démographique de la province étaient attribuables à l'accroissement naturel (Landau et Gindrey $2008: 6$ et 11$)$.

3. Ce processus de gentrification se traduit notamment par l'établissement croissant d'immeubles résidentiels, de restaurants, de bars et de centres culturels qui véhiculent une image à la fois de luxe et d'innovation artistique. Le centre culturel Arts on Main, un espace d'exposition du Centre culturel allemand qui y est associé, le centre Main Street Life comprenant des studios d'artiste, un hôtel, un cinéma, des magasins et des appartements, en sont des exemples.

4. Entre le 11 et le 23 mai 2008 des émeutes violentes ont visé majoritairement des étrangers dans la plupart des townships et des zones urbaines informelles des grandes villes de l'Afrique du Sud, faisant 62 morts, 670 blessés graves et 17000 personnes déplacées selon la police ou 
200000 selon les associations de défense des droits des migrants (CoRMSA 2008; Polzer et Igglesdon 2009).

5. Dans la deuxième moitié de l'année 2009 , le taux de chômage officiel était de $24,5 \%$.

6. Il s'agit d'un corpus de 46 entretiens semi-directifs, menés par le Forced Migration Studies Programme à l'université du Witwatersrand à Johannesburg dans les mois qui ont suivi les émeutes de mai 2008.

7. Ces recherches, non publiées jusqu'à présent, ont été menées par Elise Palomares dans le cadre du projet ANR Mitrans mentionné plus haut.

8. J'utilise la graphie «représentation(s)» pour souligner le double sens de "représentations» (Vorstellungen) et de "présentation » (Darstellung) du concept et pour renvoyer au concept de représentation(s) que j’ai développé (Ludl 2008a, 2008b).

9. La vallée du fleuve Sénégal est une région frontalière entre le Mali, le Sénégal et la Mauritanie, où l'on trouve deux groupes ethniques principaux, les Soninke et les Haalpulaar. Cette région se caractérise par des migrations anciennes et fournit le gros des migrants subsahariens en France (voir Manchuelle 1997 ; Bâ 1996 ; Gonin 2005 ; Daum 1998 ; Timera 1996 ; Lima 2003 ; Quiminal 1991). Les migrants venant d'autres régions du Sénégal concernent surtout les migrations mourides (voir Cruise 1975 ; Diouf 2000 ; Coulon 1981 ; Coulon et Cruise 1988 ; Copans 1989 ; Bava 2000, 2002 ; Bava et Gueye 2001; Buggenhagen 2002, 2004 ; Tall 1998 ; Schmidt Di Friedberg et Blion 2000 ; Riccio 2006 ; Faye 2001 ; Carter 1997). Sur la Tijaniyya, voir par exemple Triaud 2000. Contrairement aux nombreux travaux sur les migrations des deux groupes en Europe et aux États-Unis, il existe très peu d'études sur les migrants ouest-africains en Afrique du Sud en général, et sur des migrants sénégalais et maliens en particulier (Bouillon 1999; Lekogo 2006; Fall 2004 ; Vigouroux 2005 et 2008 ; Lliteras 2009).

10. L'enquête s'appuie sur une recherche de terrain menée entre avril 2009 et mai 2010 dans le centre-ville de Johannesburg (Yeoville, Bezuidenhout Valley, Ellis Park, Troyeville et le Central Business District $[\mathrm{C} B \mathrm{BD}]$ ) et sur des marchés touristiques à Johannesburg et dans ses environs. Elle a combiné des entretiens non-directifs avec une enquête ethnographique (observations et discussions dans les lieux de travail des migrants ou dans leurs maisons lors de repas ou de rassemblements religieux). Les quartiers ont été choisis selon les principaux lieux de résidence et de travail des migrants. En total, 23 entretiens ont été menés ; ils ont été enregistrés, retranscrits et analysés selon la méthode développée par Guy Michelat (1975; sur l'entretien non-directif, voir Rogers 1945 ; Donegani et al. 2002 ; Duchesne 2000, 1996 ; Duchesne et Haegel 2005 ; Martin 1999 ; Martin et Ebrahim-Vally 2006). Pour garder l'anonymat de mes interlocuteurs, j'ai remplacé leurs noms par des pseudonymes.

11. Sur les marchés touristiques à Johannesburg, voir Wa Kabwe-Segatti 2009.

12. Cassirer et Mannoni diffèrent cependant légèrement quant aux mécanismes concrets de cette transformation. Voir Ludl 2008a.

13. Sur cette constante dans les définitions de l'imaginaire, voir Castoriadis 1999: 200-201; Feron et Hastings $2002: 18$; Taylor $2004: 23-24$; Turner $1977: 71$ et $1992: 101$; Geertz 1993b : 93 ; Geertz 1993a ; Schiffauer 2006 ; Mannoni 1969. Le fait que pour la quasi-totalité des migrants que j'ai rencontrés, l'Afrique du Sud constitue une deuxième, troisième ou quatrième destination après des séjours dans d'autres pays africains, en Asie ou, bien que moins fréquemment, en Europe renvoie à cette recherche d'un idéal et des conditions tout aussi idéales pour les réaliser. Certains ont par exemple passé plusieurs années en Italie ou en Espagne, au Singapour, à Dubaï, à Bangkok, ou sont arrivés en Afrique du Sud via le Kenya et le Mozambique.

14. Cet argument a souvent fait l'objet d'interprétations culturalistes, notamment en ce qui concerne les migrants originaires de la vallée du fleuve Sénégal (N’Dongo 1975: 53, cité par Adams 1977 : 14 ; Barou 2001 ; Deniau 1996, cité par Daum 1998 : 91-92).

15. La notion de subjectivation renvoie ici à Michel Foucault et à son utilisation dans le cadre des études africaines françaises dans le sens d'une " "production de modes d'existence ou styles de 
vie" " (Deleuze 1990 : 156, commentant Foucault, cité par Bayart 1996: 157 ; voir Bayart 2004, 2006 ; Bayart et Warnier 2004).

16. Voir aussi un article paru dans la presse sénégalaise qui traite de la situation des migrants sénégalais à Grahamstown. Ces migrants déplorent de n'avoir aucune représentation des autorités sénégalaises à proximité. Au cours de l'article, on apprend qu'ils souhaiteraient cette représentation de leur pays d'origine pour obtenir des passeports dont ils ont besoin pour pouvoir se marier en Afrique du Sud et pour pouvoir voyager ensuite (Faye 2008).

17. Les chiffres proviennent de deux entretiens avec des migrants sénégalais mourides. Ils se fondent sur des recensements faits au sein de la confrérie mouride en Afrique du Sud. Cependant, comme on compte parmi les migrants sénégalais en Afrique du Sud également des membres de la Tijaniyya ainsi que des migrants sans affiliation à une confrérie, leur nombre est probablement plus élevé.

18. Le fait que la majorité des nouveaux venus déposent une demande d'asile politique et que parmi les Maliens on trouve un grand nombre de jeunes récemment arrivés permet de tirer des conclusions, bien qu'approximatives, sur le nombre de Maliens qui entrent par an en l'Afrique du Sud. Le nombre total de demandeurs d'asile maliens à l'étranger en 2007 était de 1432 . L'Afrique du Sud est ainsi le $4^{\mathrm{e}}$ pays après la France ( $\left.42 \%\right)$, les États-Unis (22\%) et l'Italie (19\%) pour ces demandes d'asile. Il est intéressant à noter qu'en 2008 ce nombre a augmenté de plus de $50 \%$ (3 776 personnes). Malheureusement, le document ne donne pas le pourcentage des demandes faites en Afrique du Sud pour cette année. On note également que ce pourcentage était en dessous de un jusqu'en 2005 et a augmenté à 5,5\% en 2006 (Ballo 2009 : 118). Le même type de document pour le Sénégal ne donne pas de chiffres qui permettraient une comparaison entre les deux pays.

19. Parmi le dispositif législatif de l'apartheid qui a perfectionné la ségrégation des groupes, le Group Areas Act de 1950 a mis en place des zones résidentielles spécifiques pour les différents groups «raciaux» et entraîné des déplacements forcés de populations. En 1951, le Bantu Authorities Act crée les homelands, des zones rurales réservées aux six groupes "ethniques » africains, où ceux-ci ont vocation à résider. Le système de l'apartheid distinguait entre "Africains », « Asiatiques/Indiens », Coloureds et «Blancs ». Comme Thomas Turino l'a souligné, la catégorie de « race » ne peut servir de catégorie analytique. " "Race"is not a conceptual tool for social analysis; rather it is the subject of such analysis» (Turino 2000:120, cité par Martin 2008: 111-112; voir Gilroy $2000: 12$ ).

20. Je monterai ailleurs comment une approche en termes de représentation(s) et l'importance de la notion de la diversité s'articulent avec la notion d'un « cosmopolitisme tactique " (Landau et Freemantle 2010) qui constitue une des démarches les plus souvent utilisées dans les analyses des phénomènes migratoires en Afrique du Sud (Ludl à paraître).

21. Sur la notion de l'aventurier, voir Pian 2009, Bredeloup et Pliez 2005, et Streiff-Fénart et Poutignat 2006.

22. L'origine nationale renvoie ici surtout à des histoires, des logiques et des expériences migratoires spécifiques. Les Sénégalais affichent une identification nationale en renvoyant à des « valeurs », à une « éducation » solide, à la religion et à des intellectuels sénégalais. 


\section{RÉSUMÉS}

Cet article met en perspective deux recherches menées au sein d'un même quartier de Johannesburg, Yeoville, qui a connu des transformations sociales et urbaines majeures dans la période post-apartheid. Les nouvelles migrations africaines venues de la République démocratique du Congo, du Congo-Brazza, du Cameroun, du Sénégal, du Mali, de Côte d'Ivoire, du Ghana, du Nigeria, du Kenya, d'Ouganda, de Somalie, d'Éthiopie et d'Érythrée transitent et résident ici comme dans la majorité des autres quartiers centraux de Johannesburg. Omniprésents dans les discours et les représentations médiatiques ou dans les conversations des Sud-africains, a fortiori lors de la multiplication des violences à leur encontre en mai dernier, ces étrangers sont rendus visibles par le vêtement, les lieux de sociabilité, les étals, les enseignes et les langues qu'ils emploient. Nous proposons d'éclairer les relations entre ces migrants - et entre étrangers et sud-africains - dans un contexte urbain où la classique opposition entre établis et outsiders prend un tour d'autant plus complexe qu'une partie des migrants rencontrés envisage Johannesburg comme une étape tandis qu'une partie des autochtones sont nouveaux venus dans la ville (naguère officiellement réservée au Blancs) en provenance des campagnes et des anciens bantoustans. Menées dans le cadre du programme Mitrans, cette enquête de C. Ludl se fonde sur une démarche ethnographique, dans le prolongement de ses recherches sur les migrants venus de la Vallée du Fleuve Sénégal. Associant enquête ethnographique et entretiens nonpréstructurés, elle met au jour les représentations que Sénégalais et Maliens ont de l'Afrique du Sud, du quartier et de ses habitants, à partir desquelles ils développent des stratégies de contrôle de l'espace et des relations sociales. Tout en développant ces stratégies, certains migrants cherchent à partir ailleurs dans la poursuite d'objectifs liés à leurs représentations de la réussite.

This article compares two studies carried out in the Johannesburg suburb of Yeoville, which has undergone major urban and social transformations since apartheid was abolished. New African migrants from the Democratic Republic of the Congo, Congo-Brazzaville, Cameroon, Senegal, Mali, Côte d'Ivoire, Ghana, Nigeria, Kenya, Uganda, Somalia, Ethiopia and Eritrea live and pass through this area, as they do in most other central Johannesburg suburbs. These foreigners are omnipresent in media discussions and representations, as well as conversations between South Africans, especially after they were subject to violent attacks last May. They are set apart by their clothing, stalls, shops, languages, and the places they socialise in. The aim of this article is to shed light on the relationships between migrants - and between foreigners and South Africans in this urban context. Here, the classic opposition between insiders and outsiders is complicated by the fact that some migrants see Johannesburg as a temporary halt, and some South Africans have only recently arrived in the city (previously reserved for Whites) from the countryside and former bantustans. Ludl's article, carried out as part of the MITRANS Research Project, builds on her research on migrants from the Senegal River valley. Combining an ethnographic field study and unstructured interviews, she describes Senegalese and Malian representations of South Africa, the suburb and its residents, and how they use this information to create strategies controlling areas and social relations. Some migrants develop these strategies even as they seek to move elsewhere to pursue goals linked to their ideas of success. 
INDEX

Thèmes : migration, mobilité, intégration

Keywords : urban violence, integration, xenophobia, Mali, Senegal, Johanesburg, South Africa

Mots-clés : violence urbaine, intégration, xénophobie, Malien, Sénégalais

Index géographique : Afrique du Sud, Johanesburg

\section{AUTEUR}

CHRISTINE LUDL

Post-doctorante associée au Centre Marc Bloch (cmb-umifre 14, http://www.cmb.huberlin.de/cmb/). De septembre 2008 à février 2010, elle a été postdoctorante à

l'Université de Witwatersrand / Institut français d'Afrique du Sud à Johannesburg (Ifasumifre 25, http://www.ifas.org.za/) dans le projet ANR « Mitrans » dirigé par Aurélia Wa Kabwe-Segatti.

chludel@zedat.fu-berlin.de 